Article

\title{
Sensitivity of the Drift-Diffusion Approach in Estimating the Power Conversion Efficiency of Bulk Heterojunction Polymer Solar Cells
}

\author{
Amir Hossein Fallahpour ${ }^{1, *}$, Aldo Di Carlo ${ }^{2}$ and Paolo Lugli ${ }^{1}$ \\ 1 Department of Electrical and Computer Engineering, Technical University of Munich, 80333 Munich, \\ Germany; lugli@tum.de \\ 2 CHOSE-Centre for Hybrid and Organic Solar Energy, Department Electronic Engineering, \\ University of Rome “Tor Vergata”, 00133 Rome, Italy; aldo.dicarlo@uniroma2.it \\ * Correspondence: amir.fallahpour@tum.de; Tel.: +49-89-289-25435
}

Academic Editor: Senthilarasu Sundaram

Received: 12 January 2017; Accepted: 21 February 2017; Published: 28 February 2017

\begin{abstract}
There are numerous theoretical approaches to estimating the power conversion efficiency (PCE) of organic solar cells (OSCs), ranging from the empirical approach to calculations based on general considerations of thermodynamics. Depending on the level of abstraction and model assumptions, the accuracy of PCE estimation and complexity of the calculation can change dramatically. In particular, PCE estimation with a drift-diffusion approach (widely investigated in the literature), strongly depends on the assumptions made for the physical models and optoelectrical properties of semiconducting materials. This has led to a huge deviation as well as complications in the analysis of simulated results aiming to understand the factors limiting the performance of OSCs. In this work, we intend to highlight the complex relation between mobility, exciton dynamics, nanoscale dimension, and loss mechanisms in one framework. Our systematic analysis represents key information on the sensitivity of the drift-diffusion approach, to estimate how physical parameters and physical processes bind the PCE of the device under the influence of structure, contact, and material layer properties. The obtained results ultimately led to recommendations for putting effort into certain properties to get the most out of avoidable losses, presented the impact and importance of modification of material properties, and in particular, recommended to what degree the design of new material could improve OSC performance.
\end{abstract}

Keywords: solar cells; power conversion efficiency (PCE); modelling and simulation; drift-diffusion; organic semiconductor

\section{Introduction}

The ultimate power conversion efficiency (PCE) of a single junction solar cell is fundamentally limited by the Shockley-Queisser (SQ) limit [1]. The applicability of the SQ theory is based on the assumption that there are upper limits for many photovoltaic systems. For instance, even the crystalline silicon solar cell has a short circuit current $\left(J_{\text {sc }}\right)$ close to SQ theory. But the deviation in PCE approximation originates from the reduction in open circuit voltage $\left(V_{\text {oc }}\right)$ as a result of non-radiative Auger recombination [2]. In view of SQ theory, ultimate efficiency of about $29 \%$ estimated for the organic absorber with the bandgap of $1.8 \mathrm{eV}$ [3]. However, in the case of organic bulk heterojunction cells, the assumptions of the SQ theory are not adequate as considerably lower PCEs are reported in the literature $[4,5]$. With the aim of minimizing energetic losses, a notable effort has been made trying to maximize the PCE of organic-based solar cells [6-10]. Despite the fact that vast progress has been made in the last decade, a reliable description of how fundamental macroscopic physical processes 
limit the PCE of organic-based solar cells is still matter of debate. In several studies, the drift-diffusion method is used to understand and study one or several aspects of sub-physical processes. However, the analysis of the simulated result with this approach is complex as the simulated result highly depends on assumptions made for the physical models and input parameters.

This work aims to evaluate and extract the PCE-limiting factors of the organic solar cells (OSCs) beyond SQ limit estimation. In particular, through a systematic study based on numerical drift-diffusion calculation, we intend to clarify the complex relation between the loss mechanism, charge carrier mobility, exciton dynamics, absorber thickness, and organic material properties in one framework. This allows defining the regimes where physical parameters and physical processes dominate the losses in OSCs which ultimately clarify how different physical processes and organic material properties condition the performance of OSCs. This work offers key information about the role of physical mechanisms, estimates the ultimate attainable efficiency based on material properties, and finally recommends how the synthesis of new polymers [9] would enhance the PCE of thin-film single-junction OSCs.

Firstly, a well-known and well-reproducible structure (Indium thin oxide (ITO)/poly-3,4ethylenedioxythiophene:poly(styrenesulfonate) (PEDOT:PSS)/poly(3-ethylenedioxy)(P3HT) blended with [6,6]-phenyl C61 butyric acid methyl ester(PC60BM)/Aluminum(Al)) is considered as a reference system. However, since only the optical absorption of the organic absorber is considered as an upper limit for photo-generated carriers, the result of this systematic simulation can be directly investigated for the analysis of other material systems.

Several processes influence the performance of OSCs, such as light absorption, exciton diffusion and dissociation, free charge carrier losses, and finally, charge carrier collection at contact [11]. Thus, simplifications of such mechanisms introduce a huge inconsistency between simulation and experimental measurements. To represent a more realistic picture and in order to compare the impact of the different loss mechanisms, we performed systematic analysis for the key parameters and physical processes that play the main role in the assumed physical model.

In particular, we investigated an approach that was able to provide insight into loss mechanisms by modeling the cell from simple to more advanced theoretical calculations. We started to calculate the efficiency limits of organic bulk heterojunction solar cells by assuming extraordinary optimum material properties and ideal conditions. Then we quantified the inconsistency between theory and experiment with associated physical processes and material parameters that influence and limit the PCE of the cell. Detailed qualitative analysis of the simulated PCE map shows to what extent the structure and material properties modification of organic material could directly influence the PCE. This can be used as a guideline to simulate the device more accurately depending on opto-electrical properties of organic material in one hand, and to direct experimentalists to develop and design optimized organic material to maximize avoidable losses toward higher device performance on the other hand. The generality of our approach for investigating a typical range of physical parameters and fundamental physical processes in our calculations do not only apply the obtained results to the organic based systems but also to other novel thin-film technologies and materials such as perovskite-based solar cells.

\section{Theoretical Background and Modeling Approach}

A detailed mathematical description of the developed model is beyond the scope of this work. Here, we simplify the picture by stating the physical processes investigated in our modeling approach. A detailed description of the developed model can be found in [12].

An organic semiconductor for an active layer of solar cells mostly absorbs photons with wavelengths smaller than $850 \mathrm{~nm}$. The active layer surrounded by several layers that have a comparable dimension with the incident light wavelength. Thus, the absorption cannot reach to its maximum value since absorption and reflection can happen on any layer and interface located before and after the absorber. 
In this work, to estimate the absorbed photons, the distribution of the optical electromagnetic field in each subsequent layer of multilayer structure was derived with the transfer-matrix method (TMM). In comparison to SQ theory, TMM would offer a more reliable approximation of upper limits of the free carrier generation.

First, we assumed that each photon with energy larger than the bandgap of absorber produces one pair of free charge carriers. Next, we coupled the photo-generated free carriers to the electrical model to probe and mimic the limitations induced by the charge transport and loss mechanism. The considered device system consists of an active layer sandwiched between a hole-extracting anode and an electron-extracting cathode (see Figure 1). The active layer is treated as an effective medium where the electron and hole transport takes place at the conduction and valance band transport levels. In this approximation, interfaces between acceptor and donor materials are not introduced in the simulation and it is assumed that the blend behaves as a single homogeneous material, thus the effective electrical band gap is given by the difference between the respective transport levels. A general schematic diagram of the primary simplified developed model is shown in Figure 1b.

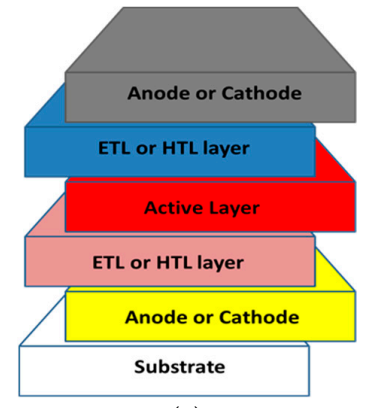

(a)

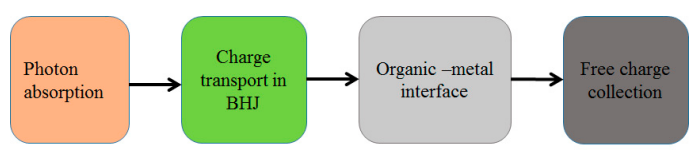

(b)

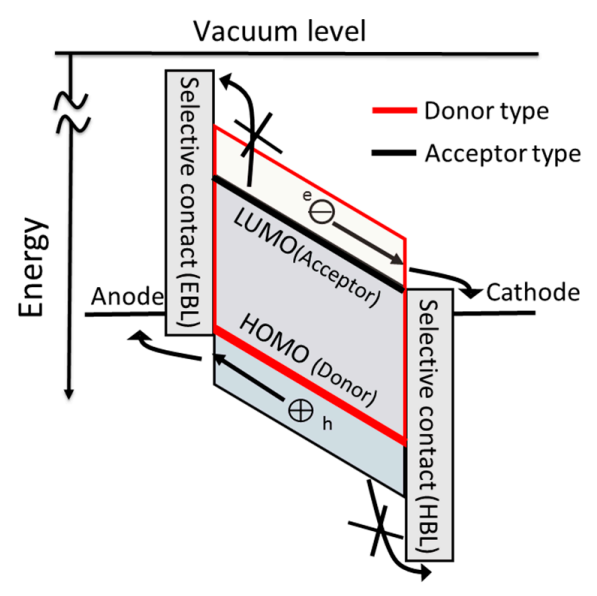

(c)

Figure 1. Schematic diagram of (a) multilayer structure; (b) primary developed model; and (c) band diagram representation of organic solar cell (OSCs). ETL: electron transport layer; HTL: hole transport layer; BHJ: bulk heterojunction; LUMO: lowest unoccupied molecular orbital; HOMO: highest occupied molecular orbital; EBL: electron blocking layer; and HBL: hole blocking layer.

Charge transport in the active layer is described by the continuity and drift-diffusion equations coupled to Poisson's equation. Alignment of the Fermi levels at the semiconductor/contact interfaces modeled by Mott-Schottky [13], and the thermionic injection model for charge carrier transfer at the metal-organic interface described through the kinetic rate model has been generalized by Scott and Malliaras [14] for the disordered materials. One of the major material properties which significantly influences the performance of OSCs is charge carrier mobility $[15,16]$. In fact, charge carrier mobility in organic materials is a function of hopping rates between donor (or acceptor) molecules that depend on the material structure and morphology of the active layer $[17,18]$. To approximate hopping mechanism in a drift-diffusion framework, a realistic model of mobility that includes the effect of electric field, temperature, and charge carrier density [19] could be investigated. In this work, as in most of the simulation approaches, the charge carrier mobilities were assumed to be a macroscopic effective parameter with a linear relation between the effective drift velocity and the electric field.

Still, there is a huge debate concerning the recombination process in organic bulk-heterojunction solar cells [20-22], however, bimolecular charge carrier recombination has been clarified using the time-of-flight method $[23,24]$. In the following sections, the role of bimolecular- and Langevin-type recombination and their effects on the performance of the cell are evaluated separately. This gives a 
comprehensive picture of the influence of charge carrier mobility, layer thickness, exciton state, and loss mechanisms on the performance of the cell. In addition, it demonstrates how PCE estimation depends on physical parameters (e.g., mobilities [25-27]) and the assumptions made for the sub-macroscopic physical processes.

\section{Ultimate PCE within Drift-Diffusion Framework}

In order to estimate the ultimate feasible PCE in the primary model, it is necessary to make several assumptions for physical models and physical parameters. To extract the maximum available photocurrent, the free charge carrier must reach the corresponding electrodes prior to recombination by driving drift or diffusion forces. In this condition, the generated free carriers can be fully utilized for photovoltaic conversion and the photocurrent of solar cells is not limited by recombination and transport loss processes. Thus, in order to maximize charge collection in related contacts, we simulated the cell assuming that the free carriers with high charge carrier mobility and without any recombination losses are transported toward the contacts. In addition, as the thermionic-diffusion injection model was considered for the charge carrier transfer at the contact, a relatively large recombination velocity was chosen for the major charge carrier to maximize the charge extraction, while inversely, a low carrier recombination velocity (selective contact) was chosen to stop the minority carrier collection at corresponding contact. The input parameters of this ideal condition are listed in Table 1.

Table 1. Basic set of parameters used for estimating ultimate power conversion efficiency (PCE) for different physical processes. N/A: not applicable.

\begin{tabular}{|c|c|c|c|c|c|}
\hline Parameters & Ultimate PCE & Bimolecular Losses & Langevin Losses & Exciton State & Unit \\
\hline Bandgap & $0.7-2$ & 1.2 & 1.2 & 1.2 & $\mathrm{eV}$ \\
\hline LUMO & -3.8 & -3.8 & -3.8 & -3.8 & $\mathrm{eV}$ \\
\hline HOMO & $-4.5--5.8$ & -5 & -5 & -5 & $\mathrm{eV}$ \\
\hline Effective density & $10^{21}$ & $10^{21}$ & $10^{21}$ & $10^{21}$ & $\mathrm{~cm}^{-3}$ \\
\hline Permittivity & 3.5 & 3.5 & 3.5 & 3.5 & - \\
\hline Exciton separation rate & $\mathrm{N} / \mathrm{A}$ & $\mathrm{N} / \mathrm{A}$ & $\mathrm{N} / \mathrm{A}$ & 0.55 to 1 & - \\
\hline Exciton decay rate & $\mathrm{N} / \mathrm{A}$ & $\mathrm{N} / \mathrm{A}$ & $\mathrm{N} / \mathrm{A}$ & 0.45 to 0 & - \\
\hline Recombination velocity & $10^{8}(\sim \infty)$ & $10^{8}$ & $10^{8}$ & $10^{8}$ & $\mathrm{~m} \cdot \mathrm{s}^{-1}$ \\
\hline Electron mobility & $100(\sim \infty)$ & $10^{-10}-100$ & $10^{-10}-100$ & $10^{-10}-100$ & $\mathrm{~m}^{2} / \mathrm{V} \cdot \mathrm{s}$ \\
\hline Hole mobility & $100(\sim \infty)$ & $10^{-10}-100$ & $10^{-10}-100$ & $10^{-10}-100$ & $\mathrm{~m}^{2} / \mathrm{V} \cdot \mathrm{s}$ \\
\hline Recombination factor & N/A & $10^{-16}-10^{-6}$ & $10^{-16}-10^{-6}$ & $10^{-16}-10^{-6}$ & $\mathrm{~cm}^{3} \cdot \mathrm{s}^{-1}$ \\
\hline Active layer thickness & $60-320$ & 200 & 200 & 200 & $\mathrm{Nm}$ \\
\hline
\end{tabular}

Typically, it is expected that increasing the thickness of the active layer enhances the photon absorption, which has a direct influence on $J_{\mathrm{sc}}$ of the cell. However, due to interference effect of the multilayer structure (inset of Figure 2), TMM simulation shows fluctuations in the absorbed photon for various thicknesses [28]. On the other hand, the opto-electrical properties of the organic semiconductor confirm the importance of the absorber bandgap. A larger bandgap is more desirable for higher $V_{\mathrm{oc}}$, while the smaller bandgap is preferred for higher $J_{\mathrm{sc}}$ (as a consequence of greater absorption). Thus, considering the assumption that we made for the physical mechanisms and parameters, absorber thickness vs. band gap was systematically simulated in order to determine the ultimate attainable PCE of the cell. Lowest unoccupied molecular orbital (LUMO) of the active layer was set to its typical value $(-3.8 \mathrm{eV})$ and highest occupied molecular orbital (HOMO) varied from $-4.5 \mathrm{eV}$ to $-5.6 \mathrm{eV}$ to reproduce the typical bandgap ranges reported for the organic active layer material $(0.7-1.8 \mathrm{eV})$. The maximum efficiency considering $200 \mathrm{~nm}$ of absorber thickness of the two well-known active layers, poly[4,8-bis[(2-ethylhexyl)oxy]benzo [1,2-b:4,5-b']dithiophene-2,6-diyl][3-fluoro-2-[(2-ethylhexyl)carbonyl]thieno[3,4-b]-thiophenediyl] (PTB7) and [6,6]-phenyl C71 butyric acid methyl ester (PC71BM) (PTB7:PC ${ }_{70} \mathrm{BM}$ ) and P3HT:PC 60 BM, was estimated to be 31.186 and 22.83, respectively. The higher PCE observed for the low bandgap PTB7:PC $70 \mathrm{BM}$ system in comparison to the $\mathrm{P} 3 \mathrm{HT}: \mathrm{PC}_{60} \mathrm{BM}$ system is due to better absorption efficiency and a broader absorption spectrum. Note that the efficiency is overestimated by several assumptions such as ultimate charge carrier mobility, no recombination processes, etc. For typical active layers (60-350) and for the nominal bandgap (1.2 eV), the maximum efficiency was estimated to be $15.509 \%$ 
for $220 \mathrm{~nm}$ and $22.36 \%$ for 260-nm active layer thicknesses for P3HT:PC 60 BM and PTB7:PC $70 \mathrm{BM}$ ,respectively (see Figure 2), representing more reliable result in compared to the overestimated PCE calculated by SQ and in [3]. In particular, a significant PCE reduction (more than $50 \%$ deviated from SQ limit for the P3HT:PC ${ }_{60} \mathrm{BM}$ system) relates to imperfect absorption of the thin-film active layer. Accordingly, the ultimate PCE estimation with this approach exhibits a proper starting point to evaluate more accurately the electrical loss mechanism of free charge carriers during their transport toward the contacts.

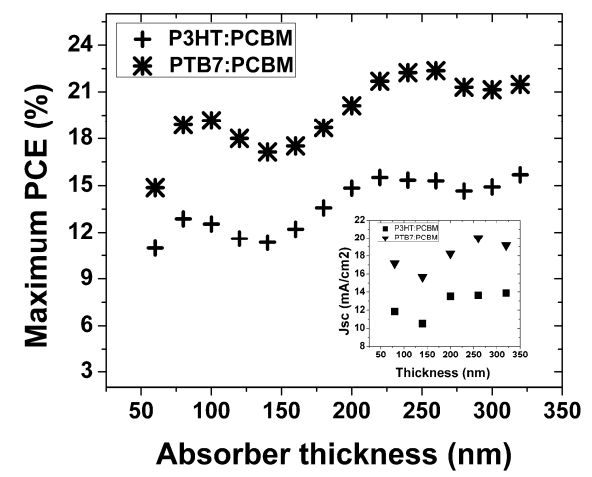

Figure 2. The final PCE estimation of conventional solar cells based on P3HT:PC ${ }_{60} \mathrm{BM}$ and PTB7:PC ${ }_{70}$ BM active layers for nominal $(1.2 \mathrm{eV})$ fixed band gap (inset: $J_{\mathrm{sc}}$ vs. active layer thickness). $J_{\mathrm{sc}}$ : short circuit current.

\section{Results and Discussion}

\subsection{Loss Mechanism and Mobility Effect}

The mobility effect on PCE of OSCs is significant, as it is correlated to the equilibrium between generation, transport, recombination, and extraction of charge carrier in the certain built-in electric field. In order to determine the optimum mobility for OSC, several theoretical studies drive the impact of mobility on device performance [26,27,29-33]. Concerning the Langevin theory, charge carrier mobility directly competes with the recombination, where the recombination increased for higher mobility. However, there is no evidence of Langevin recombination in experimental data of small molecule solar cells [34] where carrier density-dependent losses (bimolecular type) are a dominant loss mechanism [35]. Therefore, concerning thin-film low mobility absorbers, the exact role of carrier mobility and its correlation with recombination losses in the cell performance is not clear. Henceforth, we analyze both loss mechanisms individually in the following section. In this work, trap-assisted recombination losses are neglected, since such a losses mechanisms dominate only at the low light intensities [36].

\subsubsection{Ultimate PCE Map for the Carrier Density-Dependent (Bimolecular-Type) Loss Mechanism}

It has been shown in the literature that the charge recombination is sufficiently low to limit charge collection in short circuit conditions [37], but it might be a limiting factor for the PCE in the practical operation mode (maximum power point) of the cell [38]. In Section 3, to estimate the maximum achievable efficiency, the recombination process during charge transport of free carriers toward the contacts was neglected. Advancing in the study of the influence of free carrier recombination losses on the PCE, in a similar manner to the previous section we assumed that long carrier lifetime with high concentrations of charge is available after photon absorption. To minimize the losses due to surface recombination, firstly, proper recombination velocities (selective electrode) investigated at the contact allowed only one carrier type to be extracted while the extraction of the other type was prevented. In reality, however, impurity-induced recombination centers are present to some extent and effectively, nonzero surface recombination velocities exist depending on processing conditions. 
We then introduced the recombination of free carriers as a significant loss mechanism during charge carrier transport. In fact, a weakness of blending of donor and acceptor material is that the charge carriers may be lost via interfacial bimolecular recombination $\beta\left(n p-n_{i}{ }^{2}\right)$ where $n$ and $p$ are electron and hole density and $\beta$ is a morphology-dependent reduction factor [23,30,39-41].

As we assumed that the rate of recombination is independent of the mobility, we separated the role of carrier movement and recombination rate to extract their relative impact on the performance of the cell. In this regard, morphology-dependent recombination reduction factor $(\beta)$ and charge carrier mobilities varied, where other parameters were chosen for the nominal value for the typical OSCs (referenced in Table 1). While the assumption in the simulation might be non-physical, such that the recombination coefficient and mobility might be correlated to each other in very low mobilities, the simulated result gives us clear view to distinguishing the role of physical processes and to defining the regime to maximize attainable efficiency in OSCs.

Since charge collection at the contacts is extremely efficient, charge extraction rate is mostly determined by carrier movement (transit time) which is related to carrier mobility. This behavior is shown in Figure 3 where, for the typical active layer thickness of OSCs $(200 \mathrm{~nm})$, the $J_{\mathrm{sc}}$ reaches to its maximum for the charge carrier mobilities values close to those of inorganic (e.g., $\mathrm{Si}$ ) semiconductors or novel material such as Perovskite-based [42] absorbers. At this level, even an extremely high recombination factor $\left(10^{-6} \mathrm{~cm}^{3} \cdot \mathrm{s}^{-1}\right)$ does not significantly influence the $J_{\mathrm{sc}}$ of the cell. In other words, if the carrier lifetime is larger than the carrier transit time, all generated charges reach the electrodes and contribute to the photocurrent. Simulated $J_{\mathrm{sc}}$ and the PCE map in Figures 3 and 4 show a valuable picture for characterizing and parameterizing the device. For instance, in the case of high mobilities, it is clearly shown that while the $J_{\mathrm{sc}}$ reaches its maximum value for mobilities $>10^{-4} \mathrm{~m}^{2} \cdot \mathrm{V}^{-1} \cdot \mathrm{s}^{-1}$, the efficiency of the device is limited with the recombination process (see Figure 4 for PCE). Hence the parameterization and characterization of the solar cell is simple one-step $J-V$ fit, as it only relates to the recombination factor only.

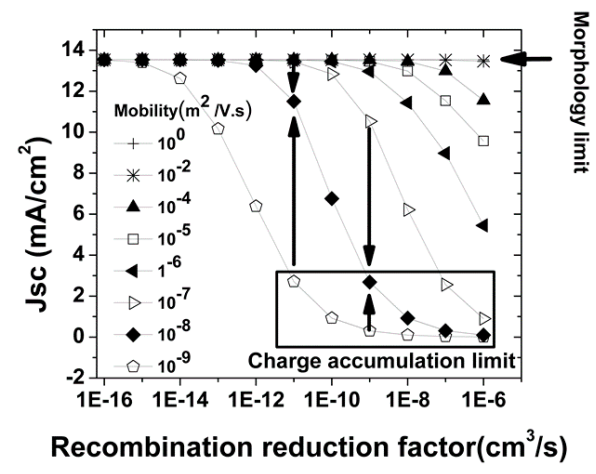

Figure 3. $J_{\mathrm{sc}}$ vs. mobility variation for different induced recombination factors.

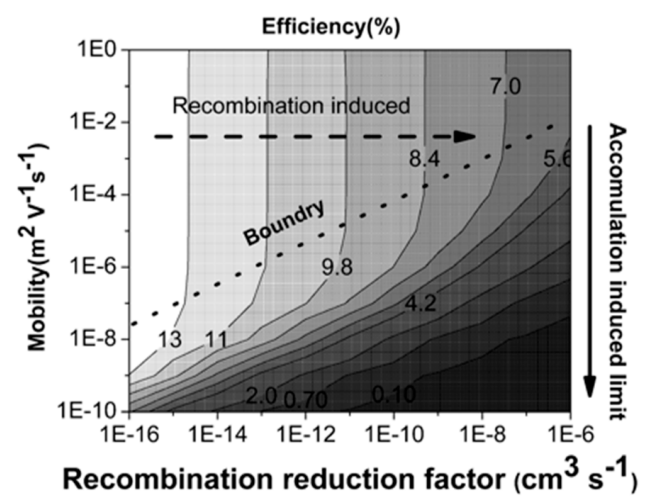

Figure 4. Contour plot presenting the ultimate achievable efficiencies for different ranges of balanced charge carrier mobilties vs. morphology-induced recombination reduction factor. 
For the lower mobilities $\left(<10^{-4} \mathrm{~m}^{2} \cdot \mathrm{V}^{-1} \cdot \mathrm{s}^{-1}\right)$ the complexity of the system is more pronounced, as the rate of free charge collection is slower than the rate of generation and transition, thus the free charges will accumulate and reach high concentrations in the device. In this regime, the recombination rate relatively increases and losses due to recombination reduce short circuit current. Unfortunately, this is a typical range of mobilities in which most of the organic semiconductors are operating in photovoltaic mode.

Figures 3 and 4 demonstrate that although the carrier mobility is reduced, the effect of recombination on PCE predominates. While orders of magnitude of recombination factor variation do not influence $J_{\mathrm{sc}}$ in the high mobility-based absorber, as shown in Figure 3, for the typical carrier mobility of conventional organic material such as P3HT:PCBM $\left(10^{-8} \mathrm{~m}^{2} \cdot \mathrm{V}^{-1} \cdot \mathrm{s}^{-1}\right)$, one order of magnitude recombination factor variation greatly influences the attainable $J_{\mathrm{sc}}$ from 11 to $2\left(\mathrm{~mA} / \mathrm{cm}^{2}\right)$. In addition, it is shown how the $J_{\mathrm{sc}}$ could jump to (or skip from) the accumulation limits for the typical recombination factors (e.g., at $10^{-11}$ and $10^{-9} \mathrm{~cm}^{3} \cdot \mathrm{s}^{-1}$ ) investigated in various OSC models.

The simulated result indicates that for inorganic or novel high mobility absorbers (e.g., perovskite), through fitting the experimental current-voltage characteristic, the recombination factor can be extracted more precisely in comparison to the complicated condition (e.g., charge accumulation effect) in a low mobility absorber system. In addition, generalized systematic simulation represents the importance and complexity of the low mobilities' correlation with the recombination process in the nanoscale devices limit.

Figure 4 represents the ultimate attainable PCE for various mobilities and recombination factor ranges. Concerning the optical absorption profile of the P3HT:PCBM, the maximum efficiency assuming high carrier mobility and low recombination rate can reach to $13.64 \%$. It is shown that for carrier mobilities higher than $10^{-2}\left(\mathrm{~m}^{2} \cdot \mathrm{V}^{-1} \cdot \mathrm{s}^{-1}\right)$ even the $J_{\mathrm{sc}}$ reaches its maximum value (see Figure 3), but the ultimate PCE of the OSC reduces to its typical reported values as a result of the recombination loss mechanism. The reduction in the efficiency could principally explain with the drops in $V_{\mathrm{oc}} . V_{\mathrm{oc}}$ corresponds the voltage at which the overall current under illumination is zero. In this condition, the photo-generation rate is balanced by recombination, where the recombination can occur either in the bulk or at the contacts as surface recombination. In this condition, $V_{\mathrm{oc}}$ is correlated to quasi-Fermi level splitting [13] and thus, as the generation rate is constant and the recombination is enhanced, lower quasi-Fermi level splitting and consequently lower $V_{\mathrm{oc}}$ will be obtained.

In particular, Figure 4 presents ultimate achievable efficiencies for different ranges of balanced charge carrier mobilities versus morphology-induced recombination losses. As depicted, for the typical carrier mobilities reported for organic semiconductors (in the range of $10^{-10}$ to $10^{-6}\left(\mathrm{~m}^{2} \cdot \mathrm{V}^{-1} \cdot \mathrm{s}^{-1}\right)$ ), charge carrier transport losses (charge accumulation) started to play a key role in cell performance. For instance, for the nominal recombination reduction factor, the efficiency of the cell improved significantly from $1.7 \%$ to $6.58 \%$ with only one order of magnitude improvement of the mobility from $10^{-9}$ to $10^{-8}\left(\mathrm{~m}^{2} \cdot \mathrm{V}^{-1} \cdot \mathrm{s}^{-1}\right)$. Such a considerable deviation in simulated results for the typical physical parameter ranges of organic semiconductors indicates why there are huge differences in the PCE estimation in the literature for the commercial low mobility-based organic absorbers such as the P3HT:PC ${ }_{60} \mathrm{BM}$-based OSCs. Specifically, the boundary (dotted line) in Figure 4 defines the regime where the transport and loss mechanisms dominate and influence the device performance. For high mobilities, the morphology-related losses (e.g., size of the island in the blend) limit the cell performance, while in low mobilities, inefficient charge transport and charge accumulation in the photoactive region are the predominant factors.

In summary, as the simulation shows, the most important limitation of PCE in organic thin film photovoltaic devices is related to low charge carrier mobility and the structural properties of disordered organic compounds which directly influence the recombination properties of the cell. Therefore, solar cell structures with enhanced carrier lifetime and higher mobilities (as long as they do not correlate to the recombination and the loss mechanism) are desired and are valuable for improving device performance. 


\subsubsection{Ultimate PCE Map for Mobility-Dependent (Lagevin Type) Losses}

In the previous section, the effect of charge carrier mobility and loss mechanisms on the PCE of the cell was analyzed through the bimolecular charge carrier density-dependent recombination process. However, in several studies, it was shown that the probability of charge carrier recombination relates to the mobility of the carrier. In fact, the expected recombination mechanism in low mobility organic materials, such as an active layer absorber of OSCs, is described by Langevin's theory [23-32] where the time for an electron and a hole to find each other defines the recombination rate. In the Langevin-type mechanism, the recombination coefficient and consequently the recombination rate is proportional to the electron and hole mobility which is described by $\gamma=\frac{\mu_{n}+\mu_{p}}{2 \varepsilon} q$. In addition, in a phase-separated blend structure, the effect of morphology leads to a reduced recombination $[23,35,40,43-47]$ which is modulated by a prefactor $\left(\beta_{L}\right)$. The reduction factor of the Langevin rate is induced (similar to bimolecular recombination coefficient) to fit and compensate the disparity between the simulation and experimental measurement. The reduction factor varies between 1 and $10^{-3}$ in OSCs [48,49], while in perovskite-based solar cells this is in the order of $10^{-5}$ [50]. In order to analyze the ultimate feasible PCE with such a recombination process, we simulated the cell by varying the main effective parameters (electron and hole mobility vs. recombination reduction factor) that concurrently influence the charge carrier transport and loss mechanism.

The $J_{\text {sc }}$ and $V_{\text {oc }}$ map for the low reduction factor $\left(\beta_{L}=10^{-6}\right)$ is shown in Figure 5a. The optimum $J_{\mathrm{sc}}$ and consequently better performance of the cell occurs once the both electron and hole mobilities are balanced. In the case of balanced mobilities (see diagonal of Figure 5a), the charge collection rate is sufficiently high to reach to its maximum value, nevertheless, as is shown in the inset of Figure 5a, through increasing the mobilities, the $V_{\mathrm{oc}}$ and relatively, the performance of the cell (Figure $5 \mathrm{~b}$ ) reduces significantly.

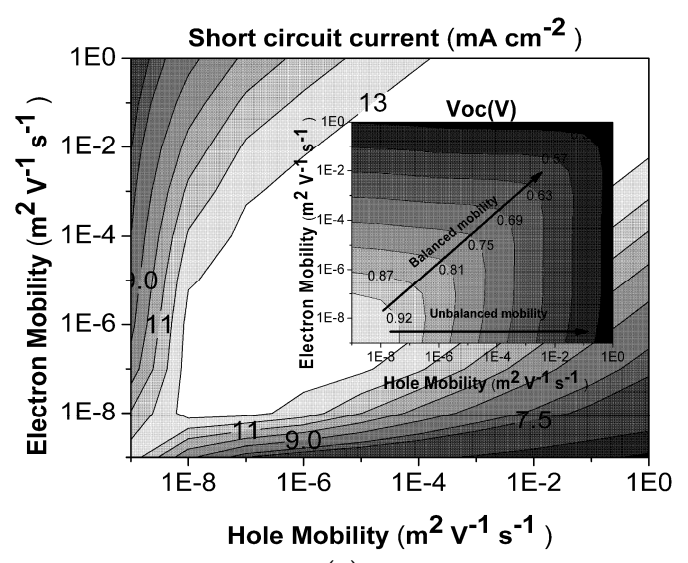

(a)

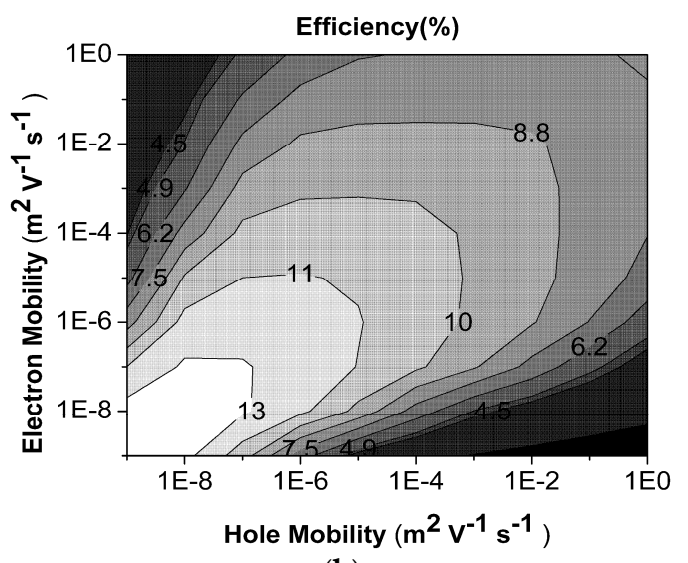

(b)

Figure 5. Counter plot showing (a) $J_{\mathrm{sc}}$, open circuit voltage $\left(V_{\mathrm{oc}}\right)$ and (b) ultimate PCE of OSC in a wide range of electron and hole charge carrier mobility variation for efficient morphology (low reduction-induced factor).

Thus, although the $J_{\mathrm{sc}}$ depends directly on the charge carrier mobility (which enhances for the higher mobilities), increasing the carrier mobility would not effectively enhance the cell performance. This is due to the fact that the recombination process is proportional to the carrier movement, hence, for high charge carrier mobility, the recombination losses increase significantly.

Note that even balanced mobilities are found to be beneficial for charge extraction; the reduction of $V_{\mathrm{oc}}$ mostly depends on one the type of carrier (see inset of Figure 5a) where the recombination constant is dominated with the faster charge carrier. In other words, even the slowest carrier mainly governs the recombination process (as the fast carrier must wait for the slow carrier to recombine), but increasing either (or both) charge carrier mobilities directly increases the rate of recombination. 
On the other hand, the reduction factor which depends on the material properties and the morphology of the absorber could have a huge influence on device performance estimation. This is shown in Figure 6, where for the same set of input parameters (see Table 1) but with a different reduction factor, the PCE of the cell was hugely influenced. For a low reduction factor, the ultimate efficiency reaches to its peak for relatively slow carrier mobilities. However, for the high reduction factor, even though the overall efficiency reduces, the maximum achievable efficiency shifts to high carrier mobilities. This is the main reason why several studies showed different values for the optimum charge carrier mobilities for the best OSC performance. For instance, there is a two order of magnitude difference in optimum mobility and a $250 \%$ PCE reduction in the ultimate attainable efficiency for the nominal and high reduction factor of organic absorbers (see Figure 6).

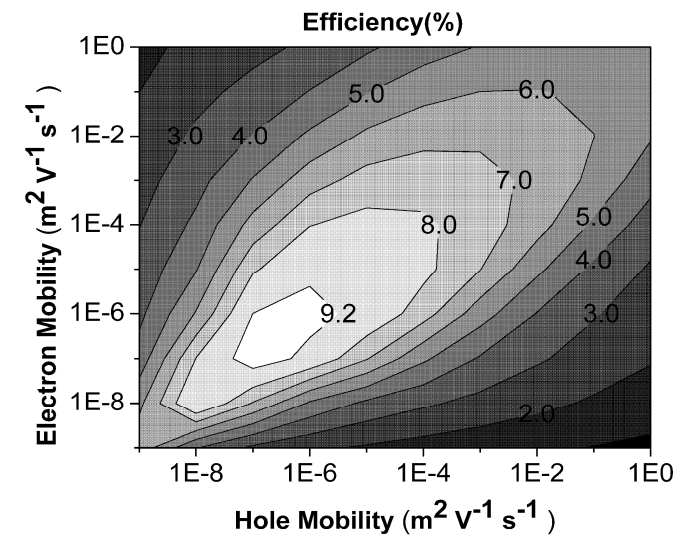

(a)

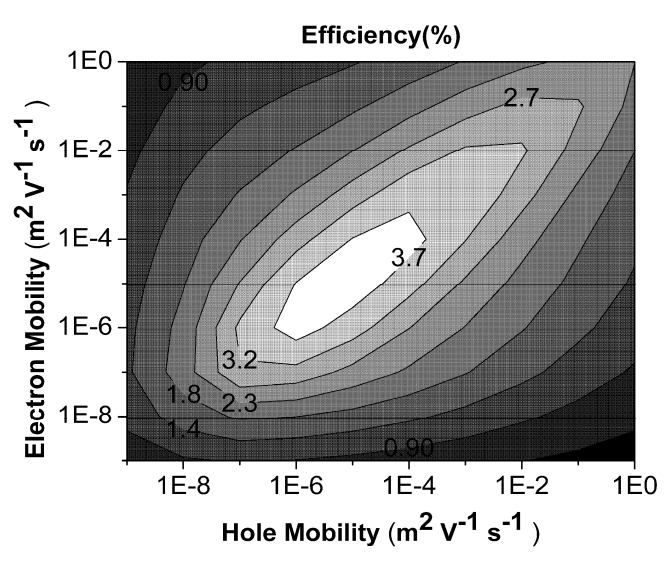

(b)

Figure 6. Counter plot showing ultimate PCE of the cell for (a) nominal and (b) high reduction factors for the same set of simulation parameter referenced in Table 1.

This parametric and systematic simulation analysis illustrates that for the typical range of carrier mobilities of organic material, the optimum efficiency was achieved only for the balanced value of mobilities, and Langevin-type recombination confirms that low charge carrier mobility is more favorable to avoid significant losses caused by the recombination losses. There are also several studies which addressed different reasons (considering different aspects) for the fact that higher mobilities may be unfavorable for device performance as a result of $V_{\text {oc }}$ reduction. For instance, authors of [26,51] showed that a reduction of $V_{\text {oc }}$ is due to recombination of dark charge carriers injected from the contacts as their density increases with the higher mobility.

On the other hand, in [31,32], the reduction of $V_{\mathrm{oc}}$ was quantified with faster charge carrier extraction as a result of high mobility. In contrast, in other studies such as that by Wagenpfahl et al. [29], the charge extraction and recombination processes were separated, assuming Langevin recombination for low mobilities and bimolecular recombination for higher mobilities. They showed that maximum efficiency can be achieved for higher mobilities.

In summary, the opto-electronic properties of the polymers are highly tunable through variation of conjugation length, varying the molecular design with new synthesis, etc. This unique property allows for designing a wide variety of material with varied opto-electrical properties that can be investigated in OSC devices [9]. Our simulation analyses elucidates the complex interplay among material properties and physical processes and it provides a guideline to recommend to what extent the material modification influences the PCE of the device.

\subsection{Role of Exciton State}

In Section 4.1, we assumed that after photon absorption, an efficient exciton separation mechanism exists in the system. Nevertheless, in organic semiconductors, absorbed photons generate an 
electron-hole pair (exciton) prior to dissociation to the free carrier. Exciton has a binding energy that is much larger than the thermal energy at room temperature. Therefore, excitons will not spontaneously split to free carrier and extra forces like band offset and energetic difference at the interface of the two organic material are required for splitting the exciton into the free carriers. The exciton-splitting mechanism has attracted notable interest and extensive studies have been dedicated to this field specifically [52,53]. Obviously, highly sophisticated and advanced models can capture the separation mechanism more accurately, however, coupling such models to the transport model adds more complexity to the system.

Several approaches attempt to combine the exciton state and charge transport with the detailed balance formalism, but none have modeled the system qualitatively to point out the limits induced by exciton dynamics. To elucidate the exciton-induced PCE limitations, we investigated a simple but straightforward approach by replacing the complex exciton model with the exciton dissociation rate. Our assumption is realistic as usually the exciton dissociation is an efficient process with marginal fluctuation $[45,54,55]$. In particular, in terms of PCE estimation, the simulated results address the relation between charge transport and exciton mechanism in one framework.

We extend the model, including the exciton state, where the excitons are assumed to dissociate with the certain probability rate. In fact, exciton losses due to inefficient exciton transport or poor exciton dissociation mechanism at the donor-acceptor interface, are replaced with the probability function model. The schematic diagram of developed model is shown in Figure 7.

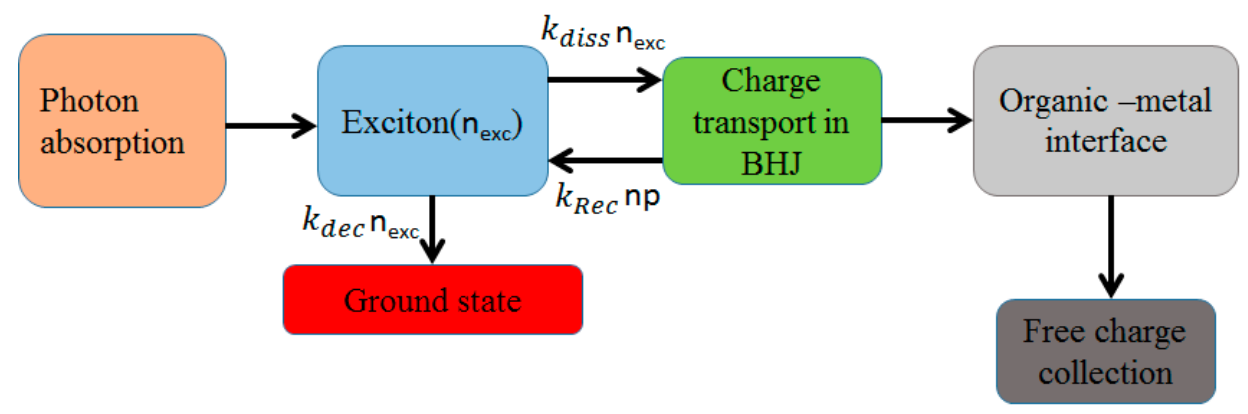

Figure 7. Schematic of the modified model by including exciton state.

In this approach, exciton density $\left(n_{\text {exc }}\right)$ is supplied through two processes assuming continuity model for exciton state. First, an absorbed photon $\left(G_{\text {optical }}(x)\right)$ directly generates an exciton, and the second process is recombination of free charge carrier $\left(G_{R(n, p)}(x)\right)$ that produces an exciton. This recombination process, coupled with the exciton state, is considered as a Langevin-type recombination. There are also two exciton state depletion processes; $k_{d e c} n_{e x c}$ and $k_{d i s s} n_{e x c} . k_{d e c}$ stands for the decay (recombination) of the exciton to the ground state and $k_{\text {diss }}$ is the rate describing the probability of exciton dissociation. Finally, this continuity equation for exciton state is coupled with drift-diffusion model.

Figure 8a shows the $J_{\mathrm{sc}}$ map of the simulated cell, as a function of exciton dissociation rate for the balanced charge mobilities (parameters are listed in Table 1). As depicted in Figure 8a, for mobilities higher than the nominal mobility of organic absorbers $\left(10^{-6}\left(\mathrm{~m}^{2} \cdot \mathrm{V}^{-1} \cdot \mathrm{s}^{-1}\right)\right)$ the $J_{\mathrm{sc}}$ tends to saturate and most of the generated free carriers are extracted efficiently. The reduction of $J_{\mathrm{sc}}$ seen for lower dissociation probability is mainly related to inefficient exciton splitting. The corresponding PCE map in this regime (Figure 8b), shows the performance of the cell saturated and further improvement of the charge carrier mobility did not effectively enhanced the performance of the device. In this regime, efficiency decreases monotonically with a nearly constant slope, independent of the mobility value. The PCE drops mainly due to reduction of photocurrent and open-circuit voltage. The former is due to lower current as a result of inefficient exciton dissociation and the latter is due to an increase of the recombination losses as a result of higher charge carrier mobilities. 


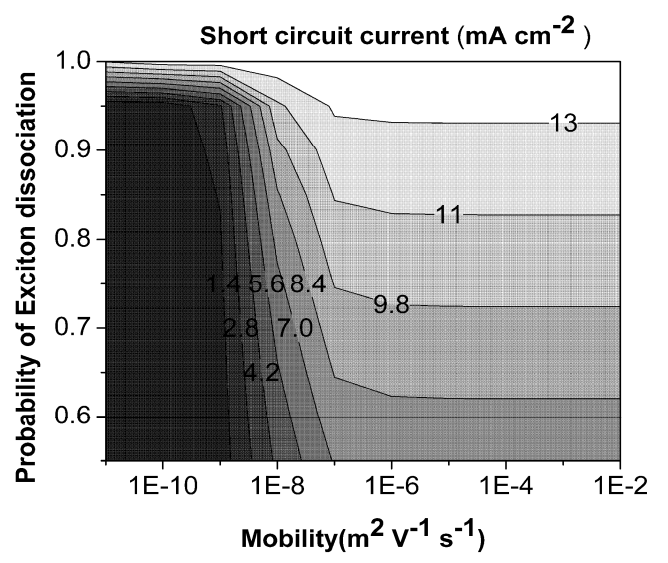

(a)

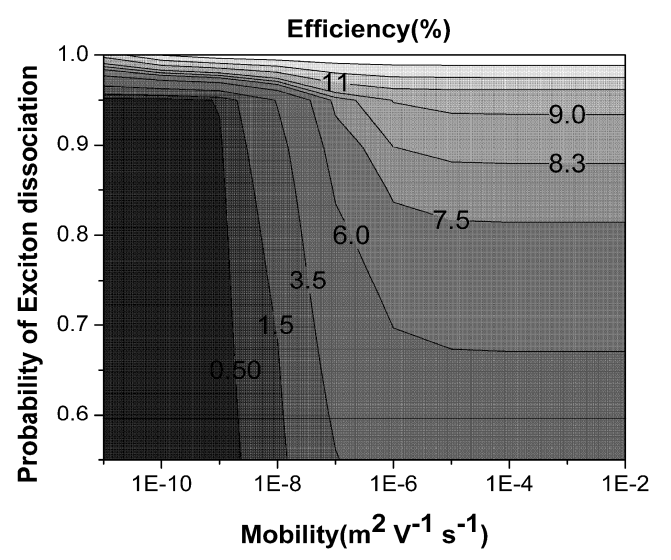

(b)

Figure 8. Counter plot of ultimate (a) $J_{\mathrm{sc}}$ and (b) PCE as a function of balanced mobility vs. exciton dissociation probability.

Completely different behavior is observed for mobilities lower than $10^{-6}\left(\mathrm{~m}^{2} \cdot \mathrm{V}^{-1} \cdot \mathrm{s}^{-1}\right)$. In this range of mobilities, not only insufficient exciton dissociation is important, but also imperfect carrier collection as a result of poor carrier transport starts to play a significant role. For instance, for the balanced mobilities, less than $10^{-8}\left(\mathrm{~m}^{2} \cdot \mathrm{V}^{-1} \cdot \mathrm{s}^{-1}\right)$, the efficiency starts to drop more rapidly in comparison to the higher mobilities with equivalent exciton dissociation probability.

This is highlighted in Figure 9 where current-voltage characteristics of low and high carrier mobilities for the efficient exciton dissociation rates are compared. The change in the performance of the device for the two orders of magnitude lower carrier mobility from $10^{-4}\left(\mathrm{~m}^{2} \cdot \mathrm{V}^{-1} \cdot \mathrm{s}^{-1}\right)$ to $10^{-6}$ $\left(\mathrm{m}^{2} \cdot \mathrm{V}^{-1} \cdot \mathrm{s}^{-1}\right)$ is very limited with a comparable fill factor (FF). However, for the mobilities close to the nominal value of conventional absorbers (e.g., $\left.10^{-8}\left(\mathrm{~m}^{2} \cdot \mathrm{V}^{-1} \cdot \mathrm{s}^{-1}\right)\right)$, the PCE and FF reduced significantly. In fact, collections of carriers become inefficient and short-circuit current, as well as the FF, start to drop dramatically. For instance, it can be stressed that for the mobility of $10^{-9}\left(\mathrm{~m}^{2} \cdot \mathrm{V}^{-1} \cdot \mathrm{s}^{-1}\right)$ which is one order of magnitude less than typical carrier mobility of $\mathrm{P} 3 \mathrm{HT}: \mathrm{PC}_{60} \mathrm{BM}$ absorber, extremely efficient exciton dissociation (not less than $96 \%$ ) is necessary and critical for efficient performance.

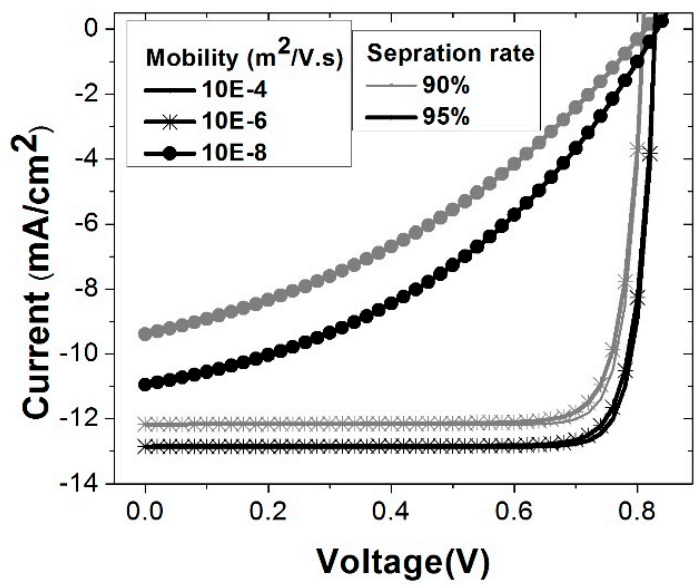

Figure 9. Current voltage characteristics of nominal and high carrier mobilities for efficient exciton separation mechanism.

This systematic simulation once more represents how critical the role of the mobility of organic materials is. As shown in Figure 8b, for even one order of magnitude higher mobilities reported for the P3HT: $\mathrm{PC}_{60} \mathrm{BM}$ system, the PCE peak is less sensitive to the charge carrier mobilities and inefficient 
exciton dissociation mechanism is a dominant limiting factor for cell performance. Thus, it is critical to develop, synthesize and process optimize an organic blend that enhances the exciton dissociation rate rather than improving the charge carrier mobilities. In contrast, for one order of magnitude lower mobilities, the role of charge carrier transport is more pronounced. In this regime, even developing heterojunction with very efficient exciton separation mechanism (higher than $90 \%$ ) is not sufficient to achieve high device performances. Therefore, for higher PCE in this boundary, it is recommendable to put effort into improving charge carrier mobility rather than optimizing the blend for a better exciton dissociation mechanism.

\section{Conclusions}

In conclusion, to estimate the ultimate attainable PCE of the OSCs beyond the SQ limits, we developed a macroscopic model from a very simple to state-of-the-art theory of organic semiconductor device physics. The developed model describes the impact of different sub-macroscopic physical processes and organic material properties on the performance of the OSC. To explain the huge gap and inconsistencies observed between the experiments and estimated ultimate attainable PCE, a different set of simulations was performed over the main physical parameters and sub-physical processes occurring in OSC. Qualitative comparison of simulated results showed the main PCE-limiting factors and recommended an alternative pathway to improve the device performance. Systematic simulation analysis showed the PCE losses in OSCs are mostly related to poor carrier transport and the recombination process. In addition, it is shown these two factors are highly correlated to each other. Hence, accounting for their relative impact on the PCE, we define a different regime where material properties and physical processes dominate the losses in OSCs. Above all, we have shown that the results of simulations and the estimation of optimum physical parameters depend strongly on the assumptions made in the physics of the device. In particular, we noted that the impact of low mobility on the PCE is considerably high and complex. Ultimately, a huge deviation and sensitivity of the simulation for a typical range of carrier mobility values reveal that to obtain reliable simulation results, precise parameterization with experimental measurements is particularly crucial for the low mobility absorbers. In addition, our comprehensive simulation study on the sub-microscopic physical processes contributes to a better understanding of the PCE-limiting factors and complexity of the parameterization and characterization of OSC for low mobilities absorbers. Finally, simulated PCE maps represent key information to predict to what extent the process, design and synthesis of the novel material and structure can enhance the performance of the solar cells toward higher efficiencies.

Acknowledgments: Amir Hossein Fallahpour and Paolo Lugli acknowledge the "LORIX-Large Organic Robust Imager for X-ray Sensing (HORIZON 2020-N644103)" Project for financial support.

Author Contributions: Amir Hossein Fallahpour developed the model and performed opto-electrical simulations. Amir Hossein Fallahpour and Paolo Lugli supervised and analyzed the methodologies and the achieved results. All authors contributed to writing and reviewing the manuscript.

Conflicts of Interest: The authors declare no conflict of interest.

\section{References}

1. Shockley, W.; Queisser, H.J. Detailed balance limit of efficiency of $p$ - $n$ junction solar cells. J. Appl. Phys. 1961, 32, 510-519. [CrossRef]

2. Green, M.A. Limits on the open-circuit voltage and efficiency of silicon solar cells imposed by intrinsic auger processes. IEEE Trans. Electron. Devices 1984, 31, 671-678. [CrossRef]

3. Kirchartz, T.; Taretto, K.; Rau, U. Efficiency limits of organic bulk heterojunction solar cells. J. Phys. Chem. C 2009, 113, 17958-17966. [CrossRef]

4. Green, M.A.; Emery, K.; Hishikawa, Y.; Warta, W.; Dunlop, E.D. Solar cell efficiency tables (Version 48). Prog. Photovolt. Res. Appl. 2016, 24, 905-913. [CrossRef]

5. Scharber, M.C.; Sariciftci, N.S. Efficiency of bulk-heterojunction organic solar cells. Prog. Polym. Sci. 2013, 38, 1929-1940. [CrossRef] [PubMed] 
6. Zhao, W.; Qian, D.; Zhang, S.; Li, S.; Inganäs, O.; Gao, F.; Hou, J. Fullerene-free polymer solar cells with over 11\% efficiency and excellent thermal stability. Adv. Mater. 2016, 28, 4734-4739. [CrossRef] [PubMed]

7. Green, M.A.; Emery, K.; Hishikawa, Y.; Warta, W.; Dunlop, E.D. Solar cell efficiency tables (Version 47). Prog. Photovolt. Res. Appl. 2016, 24, 3-11. [CrossRef]

8. He, Z.; Xiao, B.; Liu, F.; Wu, H.; Yang, Y.; Xiao, S.; Wang, C.; Russell, T.P.; Cao, Y. Single-junction polymer solar cells with high efficiency and photovoltage. Nat. Photonics 2015, 9, 174-179. [CrossRef]

9. Lu, L.; Zheng, T.; Wu, Q.; Schneider, A.M.; Zhao, D.; Yu, L. Recent advances in bulk heterojunction polymer solar cells. Chem. Rev. 2015, 115, 12666-12731. [CrossRef] [PubMed]

10. Fallahpour, A.H.; Ulisse, G.; Auf der Maur, M.; Di Carlo, A.; Brunetti, F. 3-D simulation and optimization of organic solar cell with periodic back contact grating electrode. IEEE J. Photovolt. 2015, 5, 591-596. [CrossRef]

11. Nayak, P.K.; Garcia-Belmonte, G.; Kahn, A.; Bisquert, J.; Cahen, D. Photovoltaic efficiency limits and material disorder. Energy Environ. Sci. 2012, 5, 6022-6039. [CrossRef]

12. Fallahpour, A.H.; Gagliardi, A.; Santoni, F.; Gentilini, D.; Zampetti, A.; Auf der Maur, M.; Di Carlo, A. Modeling and simulation of energetically disordered organic solar cells. J. Appl. Phys. 2014, 116, 184502. [CrossRef]

13. Zampetti, A.; Fallahpour, A.H.; Dianetti, M.; Salamandra, L.; Santoni, F.; Gagliardi, A.; Auf der Maur, M.; Brunetti, F.; Reale, A.; Brown, T.M.; et al. Influence of the interface material layers and semiconductor energetic disorder on the open circuit voltage in polymer solar cells. J. Polym. Sci. Part B Polym. Phys. 2015, 53, 690-699. [CrossRef]

14. Scott, J.C.; Malliaras, G.G. Charge injection and recombination at the metal-organic interface. Chem. Phys. Lett. 1999, 299, 115-119. [CrossRef]

15. Neher, D.; Kniepert, J.; Elimelech, A.; Koster, L.J.A. A new figure of merit for organic solar cells with transport-limited photocurrents. Sci. Rep. 2016, 6, 24861. [CrossRef] [PubMed]

16. Bartelt, J.A.; Lam, D.; Burke, T.M.; Sweetnam, S.M.; McGehee, M.D. Charge-carrier mobility requirements for bulk heterojunction solar cells with high fill factor and external quantum efficiency $>90 \%$. Adv. Energy Mater. 2015, 5, 1500577. [CrossRef]

17. Coehoorn, R.; Pasveer, W.F.; Bobbert, P.A.; Michels, M.A.J. Charge-carrier concentration dependence of the hopping mobility in organic materials with gaussian disorder. Phys. Rev. B 2005, 72, 155206. [CrossRef]

18. Tessler, N.; Preezant, Y.; Rappaport, N.; Roichman, Y. Charge transport in disordered organic materials and its relevance to thin-film devices: A tutorial review. Adv. Mater. 2009, 21, 2741-2761. [CrossRef]

19. Pasveer, W.F.; Cottaar, J.; Tanase, C.; Coehoorn, R.; Bobbert, P.A.; Blom, P.W.M.; de Leeuw, D.M.; Michels, M.A.J. Unified description of charge-carrier mobilities in disordered semiconducting polymers. Phys. Rev. Lett. 2005, 94, 206601. [CrossRef] [PubMed]

20. Proctor, C.M.; Kuik, M.; Nguyen, T.-Q. Charge carrier recombination in organic solar cells. Prog. Polym. Sci. 2013, 38, 1941-1960. [CrossRef]

21. Bergqvist, J.; Tress, W.; Forchheimer, D.; Melianas, A.; Tang, Z.; Haviland, D.; Inganäs, O. New method for lateral mapping of bimolecular recombination in thin-film organic solar cells. Prog. Photovolt. Res. Appl. 2016, 24, 1096-1108. [CrossRef]

22. Paulke, A.; Stranks, S.D.; Kniepert, J.; Kurpiers, J.; Wolff, C.M.; Schön, N.; Snaith, H.J.; Brenner, T.J.K.; Neher, D. Charge carrier recombination dynamics in perovskite and polymer solar cells. Appl. Phys. Lett. 2016, 108, 113505. [CrossRef]

23. Pivrikas, A.; Juška, G.; Mozer, A.J.; Scharber, M.; Arlauskas, K.; Sariciftci, N.S.; Stubb, H.; Österbacka, R. Bimolecular recombination coefficient as a sensitive testing parameter for low-mobility solar-cell materials. Phys. Rev. Lett. 2005, 94, 176806. [CrossRef] [PubMed]

24. Shuttle, C.G.; O’Regan, B.; Ballantyne, A.M.; Nelson, J.; Bradley, D.D.C.; Durrant, J.R. Bimolecular recombination losses in polythiophene: Fullerene solar cells. Phys. Rev. B 2008, 78, 113201. [CrossRef]

25. Koster, L.J.A. Charge carrier mobility in disordered organic blends for photovoltaics. Phys. Rev. B 2010, 81, 205318. [CrossRef]

26. Shieh, J.-T.; Liu, C.-H.; Meng, H.-F.; Tseng, S.-R.; Chao, Y.-C.; Horng, S.-F. The effect of carrier mobility in organic solar cells. J. Appl. Phys. 2010, 107, 084503. [CrossRef]

27. Tress, W.; Leo, K.; Riede, M. Optimum mobility, contact properties, and open-circuit voltage of organic solar cells: A drift-diffusion simulation study. Phys. Rev. B 2012, 85, 155201. [CrossRef] 
28. Fallahpour, A.H.; Gentilini, D.; Gagliardi, A.; Auf der Maur, M.; Lugli, P.; Di Carlo, A. Systematic study of the PCE and device operation of organic tandem solar cells. IEEE J. Photovolt. 2016, 6, 202-210. [CrossRef]

29. Wagenpfahl, A.; Deibel, C.; Dyakonov, V. Organic solar cell efficiencies under the aspect of reduced surface recombination velocities. IEEE J. Sel. Top. Quantum Electron. 2010, 16, 1759-1763. [CrossRef]

30. Kirchartz, T.; Pieters, B.E.; Taretto, K.; Rau, U. Mobility dependent efficiencies of organic bulk heterojunction solar cells: Surface recombination and charge transfer state distribution. Phys. Rev. B 2009, 80, 035334. [CrossRef]

31. Mandoc, M.M.; Koster, L.J.A.; Blom, P.W.M. Optimum charge carrier mobility in organic solar cells. Appl. Phys. Lett. 2007, 90, 133504. [CrossRef]

32. Deibel, C.; Wagenpfahl, A.; Dyakonov, V. Influence of charge carrier mobility on the performance of organic solar cells. Phys. Status Solidi Rapid Res. Lett. 2008, 2, 175-177. [CrossRef]

33. Albes, T.; Lugli, P.; Gagliardi, A. Investigation of the blend morphology in bulk-heterojunction organic solar cells. IEEE Trans. Nanotechnol. 2016, 15, 281-288. [CrossRef]

34. Tress, W.; Pfuetzner, S.; Leo, K.; Riede, M. Open circuit voltage and IV curve shape of ZnPc:C60 solar cells with varied mixing ratio and hole transport layer. J. Photonics Energy 2011, 1, 011114. [CrossRef]

35. Pivrikas, A.; Sariciftci, N.S.; Juška, G.; Österbacka, R. A review of charge transport and recombination in polymer/fullerene organic solar cells. Prog. Photovolt. Res. Appl. 2007, 15, 677-696. [CrossRef]

36. Tzabari, L.; Tessler, N. Shockley-read-hall recombination in P3Ht:PCBM solar cells as observed under ultralow light intensities. J. Appl. Phys. 2011, 109, 064501. [CrossRef]

37. Nelson, J. Diffusion-limited recombination in polymer-fullerene blends and its influence on photocurrent collection. Phys. Rev. B 2003, 67, 155209. [CrossRef]

38. Koster, L.J.A.; Smits, E.C.P.; Mihailetchi, V.D.; Blom, P.W.M. Device model for the operation of polymer/fullerene bulk heterojunction solar cells. Phys. Rev. B 2005, 72, 085205. [CrossRef]

39. Fallahpour, A.H.; Gagliardi, A.; Gentilini, D.; Zampetti, A.; Santoni, F.; Auf der Maur, M.; Di Carlo, A. Optoelectronic simulation and thickness optimization of energetically disordered organic solar cells. J. Comput. Electron. 2014, 13, 933-942. [CrossRef]

40. Koster, L.J.A.; Mihailetchi, V.D.; Blom, P.W.M. Bimolecular recombination in polymer/fullerene bulk heterojunction solar cells. Appl. Phys. Lett. 2006, 88, 052104. [CrossRef]

41. Christ, N.S.; Kettlitz, S.W.; Valouch, S.; Zufle, S.; Gartner, C.; Punke, M.; Lemmer, U. Nanosecond response of organic solar cells and photodetectors. J. Appl. Phys. 2009, 105, 104513. [CrossRef]

42. Motta, C.; El-Mellouhi, F.; Sanvito, S. Charge carrier mobility in hybrid halide perovskites. Sci. Rep. 2015, 5, 12746. [CrossRef] [PubMed]

43. Heiber, M.C.; Baumbach, C.; Dyakonov, V.; Deibel, C. Encounter-limited charge-carrier recombination in phase-separated organic semiconductor blends. Phys. Rev. Lett. 2015, 114, 136602. [CrossRef] [PubMed]

44. Groves, C.; Greenham, N.C. Bimolecular recombination in polymer electronic devices. Phys. Rev. B 2008, 78, 155205. [CrossRef]

45. Deibel, C. Charge carrier dissociation and recombination in polymer solar cells. Phys. Status Solidi 2009, 206, 2731-2736. [CrossRef]

46. Pivrikas, A.; Neugebauer, H.; Sariciftci, N.S. Charge carrier lifetime and recombination in bulk heterojunction solar cells. IEEE J. Sel. Top. Quantum Electron. 2010, 16, 1746-1758. [CrossRef]

47. Petersen, A.; Kirchartz, T.; Wagner, T.A. Charge extraction and photocurrent in organic bulk heterojunction solar cells. Phys. Rev. B 2012, 85, 045208. [CrossRef]

48. Wetzelaer, G.-J.A.H.; Van der Kaap, N.J.; Koster, L.J.A.; Blom, P.W.M. Quantifying bimolecular recombination in organic solar cells in steady state. Adv. Energy Mater. 2013, 3, 1130-1134. [CrossRef]

49. Murthy, D.H.K.; Melianas, A.; Tang, Z.; Juška, G.; Arlauskas, K.; Zhang, F.; Siebbeles, L.D.A.; Inganäs, O.; Savenije, T.J. Origin of reduced bimolecular recombination in blends of conjugated polymers and fullerenes. Adv. Funct. Mater. 2013, 23, 4262-4268. [CrossRef]

50. Wehrenfennig, C.; Eperon, G.E.; Johnston, M.B.; Snaith, H.J.; Herz, L.M. High charge carrier mobilities and lifetimes in organolead trihalide perovskites. Adv. Mater. 2014, 26, 1584-1589. [CrossRef] [PubMed]

51. Wang, Y.-X.; Tseng, S.-R.; Meng, H.-F.; Lee, K.-C.; Liu, C.-H.; Horng, S.-F. Dark carrier recombination in organic solar cell. Appl. Phys. Lett. 2008, 93, 163303. [CrossRef]

52. Kuzmich, A.; Padula, D.; Ma, H.; Troisi, A. Trends in the electronic and geometric structure of non-fullerene based acceptors for organic solar cells. Energy Environ. Sci. 2017, 10, 395-401. [CrossRef] 
53. Ma, H.; Troisi, A. Modulating the exciton dissociation rate by up to more than two orders of magnitude by controlling the alignment of lumo +1 in organic photovoltaics. J. Phys. Chem. C 2014, 118, 27272-27280. [CrossRef]

54. Arkhipov, V.I.; Heremans, P.; Bassler, H. Why is exciton dissociation so efficient at the interface between a conjugated polymer and an electron acceptor? Appl. Phys. Lett. 2003, 82, 4605-4607. [CrossRef]

55. Deibel, C.; Strobel, T.; Dyakonov, V. Role of the charge transfer state in organic donor-acceptor solar cells. Adv. Mater. 2010, 22, 4097-4111. [CrossRef] [PubMed]

(C) 2017 by the authors. Licensee MDPI, Basel, Switzerland. This article is an open access article distributed under the terms and conditions of the Creative Commons Attribution (CC BY) license (http://creativecommons.org/licenses/by/4.0/). 